\title{
CEACAM1 and the regulation of mucosal inflammation
}

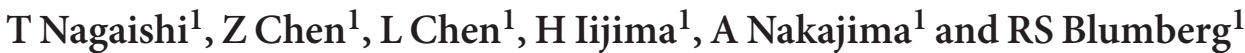

Inflammatory bowel disease (IBD) is characterized by unrestrained T-cell activation that results in the production of a variety of inflammatory cytokines and other mediators. Understanding the mechanisms of T-cell regulation is therefore of significant importance to IBD and other forms of dysregulated-mucosal inflammation. An area that is of significant interest are the cell autonomous mechanisms of T-cell regulation through proteins that have natural inhibitory functions when expressed on T lymphocytes. One such molecule is carcinoembryonic antigen cell adhesion molecule 1 (CEACAM1). CEACAM1 is primarily an activation-induced cell-surface molecule that functions as a co-inhibitory receptor. Homophilic ligation of CEACAM1 on T cells leads to a signaling mechanism, which results in inhibition of a broad range T-cell functions. CEACAM1 therefore represents a new potential therapeutic target in the treatment of IBD.

\section{INTRODUCTION}

Inflammatory bowel disease (IBD) represents a dysregulatedmucosal immune response to components of the commensal microbiota in a genetically susceptible host. ${ }^{1,2}$ This genetically determined, immunologically mediated tissue inflammation derives from excess $\mathrm{T}$ helper cytokines that are proinflammatory and which overwhelm the normal regulatory pathways of the intestines. Thus, a major focus of attention in research related to IBD derives from understanding the mechanisms of immune regulation.

A major means of regulation that has generated significant interest are those pathways that are associated with a variety of regulatory T-cell subsets that are either derived from the thymus $\left(\mathrm{CD} 4{ }^{+} \mathrm{CD} 25^{+} \mathrm{FoxP}^{+} \mathrm{T}\right.$ cells) or those FoxP ${ }^{+} \mathrm{T}$ cells that are induced within the mucosal tissues. ${ }^{3,4} \mathrm{~A}$ major feature of these cells is their ability to secrete inhibitory cytokines such as interleukin (IL)-10 and IL-35. ${ }^{5}$ It has also been recently recognized that regulation of mucosal inflammation has many additional mechanisms. For example, in addition to regulatory $\mathrm{T}$ cells, other regulatory subsets of lymphocytes have been described such as those related to $\mathrm{B}$ cells and perhaps $\mathrm{CD} 8^{+} \mathrm{T}$ cells. ${ }^{6,7} \mathrm{It}$ is also increasingly recognized that multiple layers of regulation also exist within mucosal compartments. These include those that derive from endogenous anti-inflammatory mediators as well as mediators that stimulate repair (e.g., cannabanoids, opioids, resolvins, and lipoxins), factors that block leukocyte extravasation (e.g., Apo A-IV), as well as the aforementioned inhibitory cytokines (e.g., IL-10, transforming growth factor- $\beta$, and IL-35) ${ }^{8-12}$ Moreover, it is now increasingly evident that T lymphocytes can be regulated in a cell autonomous manner through the expression of a variety of inhibitory receptors on their cell surface that include CD28-related molecules such as programmed death receptor- $1^{13}$ and, as discussed below, carcinoembryonic antigen cell adhesion molecule 1 (CEACAM1).

\section{CEACAM1}

CEACAM1 is a member of the carcinoembryonic antigen cell family of glycoproteins (Figure 1). ${ }^{14}$ These proteins consist of seven well-described family members (CEACAM1, 3-7, and 8 ) and a large number of highly related secretory molecules (pregnancy-specific glycoproteins). All of these CEACAM family members are characterized by a membrane distal IgV-related, $\mathrm{N}$-domain and variable number of IgC2-related domains. These proteins are linked to the membrane by either a glycophosphatidyl anchor (CEACAM 5, 6, 7, and 8) or by a transmembrane anchor (CEACAM1, 3, and 4). The $\mathrm{N}$-domains comprise the intercellular interaction site for homophilic and heterophilic adhesion. For example, the N-domain of CEACAM (CEA) has been shown to interact with the CD8 molecule on T lymphocytes. The $\mathrm{N}$-domain of CEACAM1 can exhibit homophilic binding with CEACAM1 as well as heterophillic binding with other CEACAM1 family members (e.g., CEACAM5 or CEA) and components of the microbial universe (e.g., murine hepatitis virus and Neisseria sp.). ${ }^{15}$ The CEACAM family members are

${ }^{1}$ Brigham and Women's Hospital, Harvard Medical School, Boston, Massachusetts, USA. Correspondence: RS Blumberg (rblumberg@partners.org) 


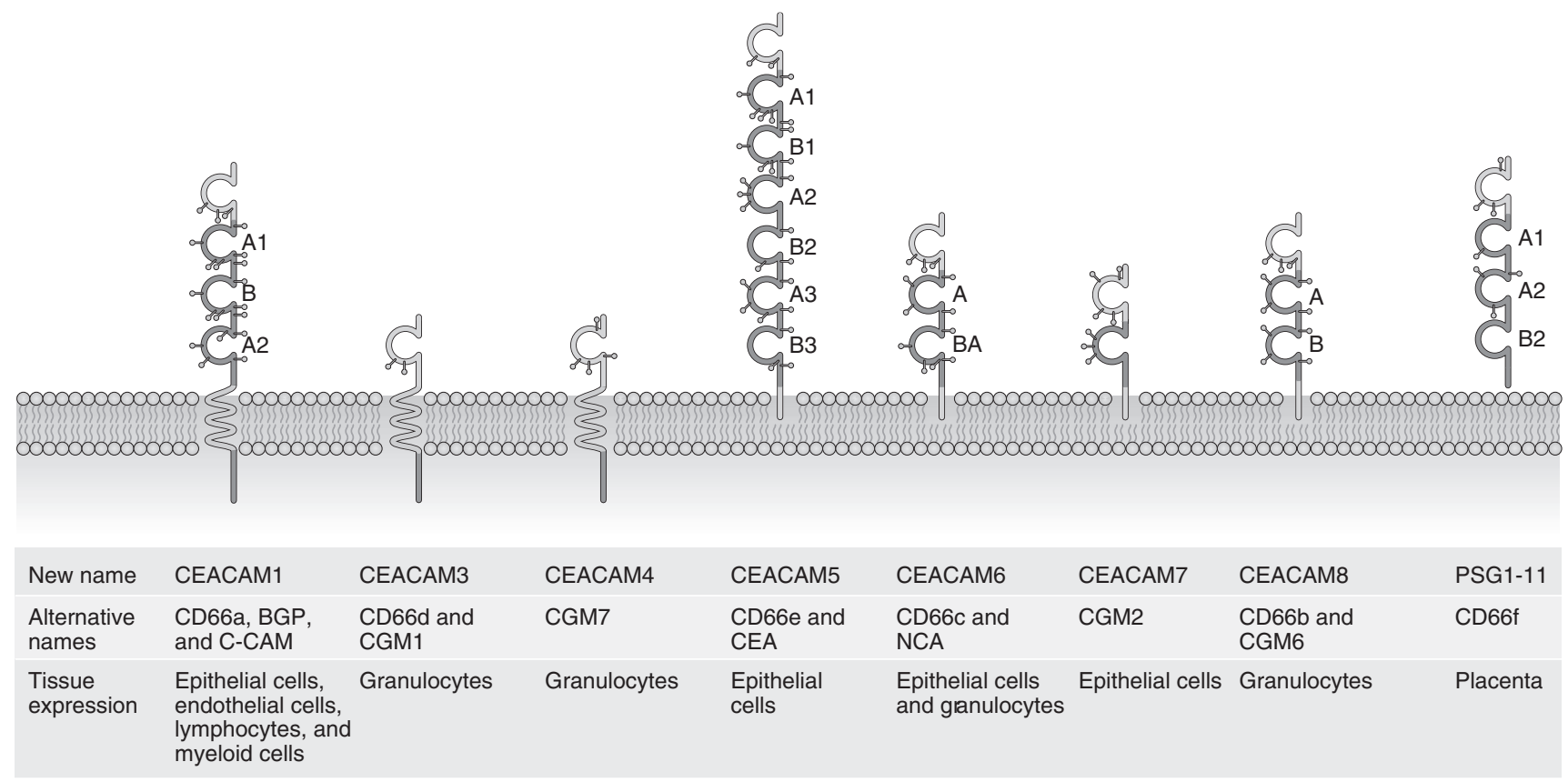

Figure 1 Summary of CEACAM family members (adapted from ref. 14). Human CEACAM family members are encoded on chromosome 19. They include the CEA-related members (CEACAM5) and pregnancy-specific glycoproteins (PSG). The latter are soluble proteins. Each member consists of a membrane distal IgV-related $\mathrm{N}$-domain and a variable number of IgGC2-related domains that are either linked to the membrane by a transmembrane anchor or glycophosphatidyl-linked anchor. Rodents (mouse and rat) only express CEACAM1. Please see the text for details.

characterized by a significant amount of alternate splicing that generates 11 different splice products in humans and four different splice products in mouse (Figure 2). These splice products are named for the number of extracellular domains and whether they have a long cytoplasmic tail with two immune receptor inhibitory motifs (ITIM) or a short new cytoplasmic tail of 10 amino acids.

\section{CEACAM1 EXPRESSION AND FUNCTION ON T LYMPHOCYTES}

CEACAM1 is expressed on a number of different cell types including epithelial cells, endothelial cells, and myeloid cells. ${ }^{14}$ It has recently been appreciated that CEACAM1 is also expressed on certain lymphocytes (B cells and natural killer cells) and on T lymphocytes primarily after activation. ${ }^{16-18}$ All T cell subsets have been shown to express CEACAM1 after activation. Low level expression of CEACAM1 can be observed on a small subset of resting CD4-positive lymphocytes in both mouse and human. However the function of this and the subset of $\mathrm{T}$ cells that express this low level of CEACAM1 has yet to be defined. As noted, CEACAM1 has multiple different splice products that are either characterized by a long or a short cytoplasmic tail. Both mouse and human T lymphocytes predominantly express isoforms with a long cytoplasmic tail, containing two ITIM domains. ${ }^{14}$ Transfection studies with human CEACAM1 isoforms have shown that those isoforms expressing a long cytoplasmic tail have an inhibitory function in T cells. ${ }^{19}$ This inhibitory function is dependent on the ITIM domains and Src homology phosphatase 1 (SHP-1). It has been shown that short cytoplasmic tail-containing isoforms possess very weak costimulatory activity, such that coexpression of these two isoforms may lead to modulation of inhibition by CEACAM1-L isoforms. Consistent with a presumed inhibitory function of CEACAM1, heterophilic ligation of mouse CEACAM1 with a monoclonal antibody to the $\mathrm{N}$-domain leads to the inhibition of a mixed lymphocyte reaction in vitro and delayed-type hypersensitivity response in hapten-mediated colitis models in vivo. ${ }^{20}$ Furthermore, overexpression of a mouse CEACAM1 isoform (CEACAM1-4L) specifically in T lymphocytes by transgenic overexpression of the CEACAM1$4 \mathrm{~L}$ isoform leads to T-cell inhibition when examined ex vivo. ${ }^{21}$ This inhibitory property of long cytoplasmic tail-containing isoforms directly affects T-cell receptor-CD3 complex signaling as shown by the inhibition of antigen-specific activation of primary CD4-positive $\mathrm{T}$ cells that are major histocompatibility complex class II (I-A ${ }^{b}$ ) and ovalbumin specific (Figure 3).

\section{REGULATION OF MUCOSAL INFLAMMATION BY CEACAM1 EXPRESSION ONT CELLS}

To determine whether CEACAM1 expression can regulate T cell-mediated inflammation, the effects of CEACAM1 ligation on T lymphocytes have been examined. In early studies, administration of a monocolonal antibody specific for the $\mathrm{N}$-domain of CEACAM1 or a Fc-fusion protein containing the $\mathrm{N}$-domain of mouse CEACAM1 to model homophilic ligation of CEACAM1 leads to amelioration of both trinitrobenzine sulfonic acid- and oxazolone-induced colitis. ${ }^{20}$ Interestingly, the protection from colitis was most prominently associated with inhibition of T helper 1 cytokines. This was shown to involve blockade of nuclear translocation of $\mathrm{T}$ box expressed in $\mathrm{T}$ cells. 


\begin{tabular}{|c|c|c|c|}
\hline Species & Gene & Splice variant & Structure \\
\hline \multirow[t]{10}{*}{ Human } & CEACAM1 & $\begin{array}{l}\text { CEACAM1-1L } \\
\text { CEACAM1-1S }\end{array}$ & $\begin{array}{l}\mathrm{N}, \mathrm{TM}, \mathrm{L} \\
\mathrm{N}, \mathrm{TM}, \mathrm{S}\end{array}$ \\
\hline & & CEACAM1-3L & N, A1, B, TM, L \\
\hline & & CEACAM1-3S & N, A1, B, TM, S \\
\hline & & CEACAM1-4L & $\mathrm{N}, \mathrm{A} 1, \mathrm{~B}, \mathrm{~A} 2, \mathrm{TM}, \mathrm{L}$ \\
\hline & & CEACAM1-4S & N, A1, B, A2, TM, S \\
\hline & & CEACAM1-3AL & N, A1, B, Alu, TM, L \\
\hline & & CEACAM1-3AS & N, A1, B, Alu, TM, S \\
\hline & & CEACAM1-3 & N, A1, B \\
\hline & & CEACAM1-4C1 & $\mathrm{N}, \mathrm{A} 1, \mathrm{~B}, \mathrm{~A} 2, \mathrm{C} 1$ \\
\hline & & CEACAM1-4C2 & $\mathrm{N}, \mathrm{A} 1, \mathrm{~B}, \mathrm{~A} 2, \mathrm{C} 2$ \\
\hline \multirow[t]{4}{*}{ Mouse } & Ceacam1 & Ceacam1-4L & $\mathrm{N}, \mathrm{A} 1, \mathrm{~B}, \mathrm{~A} 2, \mathrm{TM}, \mathrm{L}$ \\
\hline & & Ceacam1-4S & $\mathrm{N}, \mathrm{A} 1, \mathrm{~B}, \mathrm{~A} 2, \mathrm{TM}, \mathrm{S}$ \\
\hline & & Ceacam1 -2L & N, A2, TM, L \\
\hline & & Ceacam1-2S & $\mathrm{N}, \mathrm{A} 2, \mathrm{TM}, \mathrm{S}$ \\
\hline
\end{tabular}

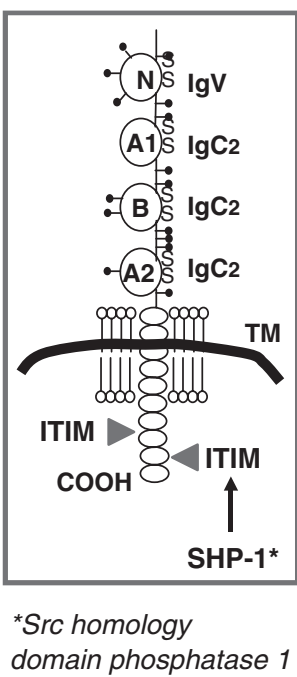

Figure 2 Summary of CEACAM1 splice products (adapted from ref. 14). The single CEACAM1 gene in humans and rodents is characterized by alternate splicing, generating 11 isoforms in humans and four isoforms in mice. The nomenclature is adapted from ref. 14 and described in the text. Splice variants that are in boxes are those that have been identified in human and mouse T cells by PCR. The schematic shows the predicted structure of a CEACAM1-4L splice variant with four extracellular domains and a long cytoplasmic tail containing two ITIM domains.

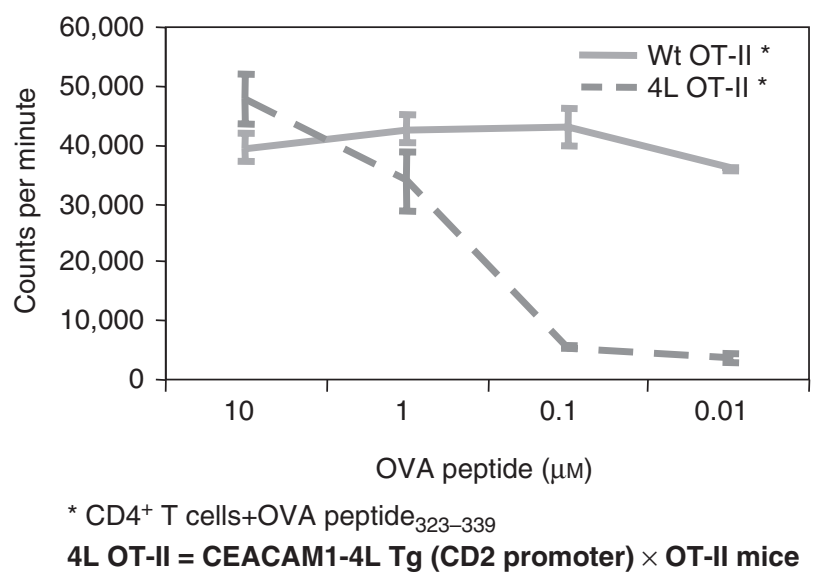

Figure 3 Inhibition of antigen-specific T-cell activation by CEACAM1 expressing a long cytoplasmic tail. CEACAM1-4L-transgenic mice (as described in ref. 21) were crossed with OT-II transgenic mice expressing a T-cell receptor specific for ovalbumin (OVA) in the context of $I-A^{d}$. T cells from these mice were exposed to different concentration of OVA in the context of wild-type antigen-presenting cells from Balb/c mice and activation assessed.

Although these studies were consistent with a model, wherein CEACAM1 expression on T cells was the mechanism for inhibition, the broad expression of CEACAM1 did not allow for a definitive conclusion that the means of inhibition was through ligation of CEACAM1 on T cells. Therefore, studies were subsequently pursued to examine the overexpression of CEACAM1 specifically on $\mathrm{T}$ cells either by retroviral infection or transgene expression. ${ }^{21}$ In the former case, it has been shown that overexpression of the CEACAM1-2L isoform in primary naive $\mathrm{T}$ cells, but not the CEACAM1-2L isoform in which the two ITIM residues were mutated, could abrogate the ability of the effector $\mathrm{T}$ cells to induce colitis after adoptive transfer into RAG2- deficient mice. These studies show that the CEACAM1-2L isoform is indeed inhibitory to T cells, and that this inhibition is dependent upon the functional signaling of the cytoplasmic tail. As the ITIM domains have been shown to mediate their inhibition through SHP-1, the role of SHP-1 in CEACAM1 signaling was examined in vivo by retrovirally infecting naive $T$ cells from SHP-1-deficient, CD4-positive T cells. In this case, the wildtype CEACAM1-2L isoform was shown to be unable to mediate inhibition in the absence of either the ITIMs or SHP-1 indicating that CEACAM1 inhibitory signaling is dependent upon the ITIMs and SHP-1 in vivo. Finally, CD4 naive, CD4-positive $T$ cells that overexpress the other long cytoplasmic tail containing type of isoform of CEACAM1 (CEACAM1-4L) in mice when overexpressed in $\mathrm{T}$ cells transgenically, also abrogated the ability of effector $\mathrm{T}$ cells to induce colitis when adoptively transferred into RAG2-deficient mice. Taken together, these studies show that indeed CEACAM1 is an inhibitory molecule in T cells and can specifically block the development of intestinal inflammation.

\section{CHARACTERIZATION OF T-CELL FUNCTION IN THE ABSENCE OF CEACAM1}

On the basis of the studies described above, it would be predicted that if CEACAM1 is an inhibitory coreceptor, it would be predicted that (deletion or loss) of CEACAM-L isoforms in T cells would increase T-cell activation. To test this, conditional deletion of CEACAM1 was accomplished by crossing mice containing lox P sites flanking exons 7 through 9 encoding the cytoplasmic tail of CEACAM1 with a mouse expressing Cre recombinase under the control of a T cell-specific promoter. ${ }^{21}$ Consistent with an inhibitory function of CEACAM1, deletion of CEACAM1 signaling function of $\mathrm{T}$ cells in this manner resulted in hyperactivation of the T cells ex vivo when stimulated with anti-CD3 and anti-CD28 as assessed by cytokine secretion. CEACAM1 has 
also been previously noted to inhibit the cytolytic function of CD8-positive T cells. Masking CEACAM1 homophilic binding on human CD8-positive cytotoxic T cells using a Fab fragment of an anti-human CEACAM1 monoclonal antibody increased the cytolytic function of the CD8-positive T cells, as assessed by lysozyme mobilization to the cell surface after activation of the T-cell receptor-CD3 complex (unpublished observations). Taken together with the overexpression studies described above, these studies with mice that are genetically deleted for CEACAM1 expression or human T cells in which CEACAM1 function has been blocked, show that CEACAM1 is indeed a co-inhibitory receptor in T cells.

In summary, CEACAM1 is a new, non-CD28-related co-inhibitory receptor that mediates inhibition of the T-cell receptor-CD3 complex. Furthermore, this inhibitory function of CEACAM1 occurs in a cell autonomous manner. These studies mark CEACAM1 as a new target for therapeutic interventions in mucosal and other types of inflammation.

\section{ACKNOWLEDGMENTS}

This study was funded by NIH grants DK44319, DK51362, DK53056, the Crohn's and Colitis Foundation of America and the Harvard Digestive Diseases Center (DK034854).

\section{DISCLOSURE}

Takashi Nagaishi is currently receiving grant support from the Japanese Ministry of Education, Culture, Sports, Science, and Technology as well as the Takeda Science Foundation, the Abbott Japan Allergy Research Award, and the Alumni Association of Nihon University School of Medicine. The remaining authors have declared no financial interests.

(C) 2008 Society for Mucosal Immunology

\section{REFERENCES}

1. Strober, W., Fuss, I.J. \& Blumberg, R.S. The immunology of mucosal models of inflammation. Ann. Rev. Immunol. 20, 495-549 (2002).

2. Xavier, R.J. \& Podolsky, D.K. Unravelling the pathogenesis of inflammatory bowel disease. Nature 448, 427-434 (2007).

3. Zheng, Y. \& Rudensky, A.Y. Foxp3 in control of the regulatory T cell lineage. Nat. Immunol. 8, 457-462 (2007).
4. Mucida, D. et al. Reciprocal TH17 and regulatory T cell differentiation mediated by retinoic acid. Science 317, 256-260 (2007).

5. Collison, L.W. et al. The inhibitory cytokine IL-35 contributes to regulatory T-cell function. Nature 450, 566-569 (2007).

6. Mizoguchi, A., Mizoguchi, E., Takedatsu, H., Blumberg, R.S. \& Bhan, A.K. Chronic intestinal inflammatory condition generates IL-10 producing regulatory B cell subset characterized by CD1d upregulation. Immunity 16, 219-230 (2002).

7. Brimnes, J., Allez, M., Dotan, I., Shao, L., Nakazawa, A. \& Mayer, L. Defects in CD8+ regulatory $T$ cells in the lamina propria of patients with inflammatory bowel disease. J. Immunol. 174, 5814-5822 (2005).

8. Kunos, G. \& Pacher, P. Cannabinoids cool the intestine. Nat. Med. 10, 678-679 (2004).

9. Phillipe, D. et al. Anti-inflammatory properties of the mu opioid receptor support its use in the treatment of colon cancer. J. Clin. Invest. 111, 1329-1338 (2003).

10. Arita, M. et al. Resolvin E1, an endogenous lipid mediator derived from omega-3 eicosapentaenoic acid, protects against 2,4,6-trinitrobenzene sulfonic acid-induced colitis. Proc. Natl. Acad. Sci. USA 102, 7671-7676 (2005).

11. Fiorucci, S. et al. A beta-oxidation-resistant A4 analog treats hapten-induced colitis by attenuating inflammation and immune dysfunction. Proc. Natl. Acad. Sci. USA 101, 15736-15741 (2004).

12. Vonwinkel, T. et al. Apolipoprotein A-IV inhibits experimental colitis. J. Clin. Invest. 114, 260-269 (2004).

13. Greenwald, R.J., Freeman, G.J. \& Sharpe, A. The B7 family revisited. Annu. Rev. Immunol. 23, 515-548 (2005).

14. Owen, G.S. \& Blumberg, R.S. Carcinoembryonic cell adhesion molecule 1 in immune regulation. Nat. Rev. Immunol. 6, 433-446 (2006).

15. Dveksler, G.S. et al. Several members of the mouse carcinoembryonic antigen-related glycoprotein family are functional receptors for the coronavirus mouse hepatitis virus A-59. J. Virol. 67, 108 (1993).

16. Markel, G. et al. Pivotal role of CEACAM1 protein in the inhibition of activated decidual lymphocyte functions. J. Clin. Invest. 110, 943-953 (2002).

17. Morales, V. et al. Regulation of human intestinal intraepithelial lymphocyte cytolytic function by biliary glycoprotein (CD66a). J. Immunol. 163, 1363-1370 (1999).

18. Nakajima, A. et al. Activation-induced expression of carcinoembryonic antigen-cell adhesion molecule 1 regulates mouse T lymphocyte function. J. Immunol. 168, 1028-1035 (2002).

19. Chen, D. et al. Carcinoembryonic antigen-related cellular adhesion molecule 1 isoforms alternatively inhibit and costimulate human T cell function. J. Immunol. 172, 3535-3543 (2004).

20. lijima, H. et al. Specific regulation of T helper 1-mediated murine colitis by CEACAM1. J. Exp. Med. 199, 471-482 (2004).

21. Nagaishi, T. et al. SHP1 Phosphatase-dependent T cell inhibition by CEACAM1 adhesion molecule isoforms. Immunity 25, 1-13 (2006). 